\title{
ORIGINAL ARTICLE \\ Antegrade continence enema procedure: impact on quality of life in patients with spinal cord injury
}

\author{
PH Smith and RM Decter
}

Study design: This is a prospective cohort study.

Objectives: Patients with spinal cord injury (SCl) often suffer from severe constipation/fecal incontinence. The antegrade continence enema (ACE) procedure is often used to control these distressing symptoms when medical management fails. Improvement in the quality of life (QOL) following the ACE procedure has been demonstrated in patients with fecal incontinence of various etiologies. We assess the impact of the ACE procedure on QOL in patients with fecal incontinence due to SCI.

Setting: This study was conducted in the United States.

Methods: We measured the impact of fecal incontinence on QOL in patients with SCl undergoing ACE using the validated fecal incontinence quality of life (FIQL) QOL instrument. The FIQL scores QOL in four domains: lifestyle, coping/behavior, depression/selfperception and embarrassment. Surveys were prospectively administered before and after surgery. Preoperative and postoperative survey scores were compared using two-sample T-test.

Results: Between 2003 and 2010, the ACE procedure was performed on 17 patients with SCl, including 10 paraplegic and seven quadriplegic patients with an average age of 33 years at the time of surgery. Scores in all four QOL realms assessed by the FIQL instrument improved significantly following the ACE procedure. Stomal stenosis requiring channel revision occurred in three patients and was the most common complication.

Conclusions: This is the first study to our knowledge that assesses the impact of the ACE procedure on the QOL in patients with SCI. Using a validated questionnaire, we demonstrated significant improvement in QOL related to fecal incontinence following the ACE procedure in these patients who are severely affected by their bowel dysfunction.

Spinal Cord (2015) 53, 213-215; doi:10.1038/sc.2014.223; published online 23 December 2014

\section{INTRODUCTION}

Neurogenic bowel dysfunction resulting in severe constipation and fecal incontinence is a very common sequela of spinal cord injury (SCI), with up to $85 \%$ of patients with SCI experiencing some degree of fecal incontinence. ${ }^{1-3}$ Severe constipation secondary to neurogenic bowel dysfunction and associated fecal incontinence has a marked impact on social and psychological well-being, and it is a source of significant distress for patients with SCI. ${ }^{2,3}$

Regular and complete colonic evacuation forms the cornerstone in the treatment of fecal incontinence due to neurogenic bowel dysfunction. The initial treatment of constipation related to neurogenic bowel dysfunction generally includes combinations of oral therapies, suppositories and enemas. Such treatments have the benefit of being noninvasive, but they may be ineffective in adequately managing the severe constipation afflicting individuals with SCI and often require the patient to rely upon the assistance of a caretaker to administer the bowel regimen.

Malone et al. ${ }^{4}$ described in 1990 the creation of an antegrade continence enema (ACE) procedure for the treatment of severe constipation. The ACE procedure allows a patient to administer an enema directly into the cecum via an easily accessible abdominal wall stoma, and it is an effective method for controlling refractory fecal incontinence due to a variety of etiologies in both adult and pediatric populations. $^{5-9}$ The ACE procedure has been shown to provide significant improvement in patient and caregiver quality of life (QOL) when used in patients with neurogenic bowel dysfunction due to a variety of etiologies; however, no study has assessed the impact of the ACE procedure on QOL in patients with neurogenic bowel dysfunction due to SCI. ${ }^{10-12}$ We use a validated QOL instrument, which specifically assesses QOL related to fecal incontinence, to objectively measure changes in QOL related to fecal incontinence before and after the ACE procedure in patients with SCI.

\section{MATERIALS AND METHODS}

By recognizing the multifactorial disability of SCI, we use the disease-specific fecal incontinence quality of life (FIQL) instrument developed by Rockwood et al. ${ }^{13}$ to specifically assess the impact of the ACE procedure on QOL related to fecal incontinence. The FIQL instrument consists of a 29-question survey that generates scores in four QOL domains: lifestyle, coping/behavior, depression/ self-perception and embarrassment. Scores for each domain are reported on a continuous scale of $1-4$, in which a higher score indicates a more favorable QOL. Rockwood et al..$^{13}$ have shown the FIQL scale to be a reliable and valid instrument to measure the QOL related to fecal incontinence. Furthermore, analysis for convergent validity demonstrates correlation of the FIQL scales with appropriately matched subscales from the SF-36 global QOL instrument, a validated and widely used instrument in studies of QOL in the SCI

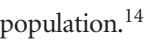


Between 2003 and 2010, FIQL surveys were administered to all patients with SCI undergoing the ACE procedure at our institution. Patients completed surveys preoperatively and postoperatively. The preoperative survey was administered during the preoperative clinic appointment, which occurred 1 to 2 weeks before the surgery date. The postoperative assessment was performed during a clinic appointment at a minimum of four months after surgery. All surveys were administered by the nurse coordinator of our neurogenic bladder/bowel clinic. In several patients in whom a preoperative FIQL could not be obtained, the preoperative survey was retrospectively completed during the postoperative period.

Data pertaining to patient age, sex, age at the time of surgery, mechanism and level of injury, type of channel (appendix or other intestinal segment), complications and length of follow-up were obtained from the medical record.

All statistical analyses were carried out using SAS statistical software version 9.3 (SAS Institute, Cary, NC, USA). Categorical data pertaining to patient characteristics, operative data and complications were summarized according to percentage and frequency. Preoperative and postoperative survey responses were compared using a paired $T$-test for the cohort as a whole, as well as separately, for the groups of patients who retrospectively or prospectively completed the preoperative evaluation. A two-sample $T$-test was used to compare the change in the survey responses between preoperative and postoperative scores.

\section{RESULTS}

Seventeen SCI patients, five female and 12 male, underwent the ACE procedure between 2003 and 2010. This group consisted of 10 paraplegic and seven quadriplegic patients. The average patient age at the time of surgery was 33 years (range 6-49). The mean duration of follow up was 56 months (range 4-102).

Five patients, who had retrospectively completed the preoperative survey after surgery, were excluded from final analysis. In the remaining 12 patients, the QOL scores increased significantly in all categories of the FIQL survey after surgery (Figure 1). The lifestyle score increased from an average of $2.3($ s.d. $=0.9)$ preoperatively to 3.7 (s.d. $=0.5)$ postoperatively $(P<0.001)$. The mean preoperative coping/ behavior score was $2.2($ s.d. $=0.9)$, versus $3.8($ s.d. $=0.3)$ postoperatively $(P<0.001)$. The mean depression/self-perception increased from 2.8 (s.d. $=0.9)$ to 3.8 (s.d. $=0.4)(P<0.001)$, and the embarrassment score improved from 2.2 (s.d. $=1.1)$ to $3.8($ s.d. $=0.3)(P<0.001)$. For all four QOL domains, the change in score from the preoperative to

\section{$\mathbb{Q}$ Preoperative Score $\square$ Postoperative Score Comparison of pre- and post-operative FIQL scores}

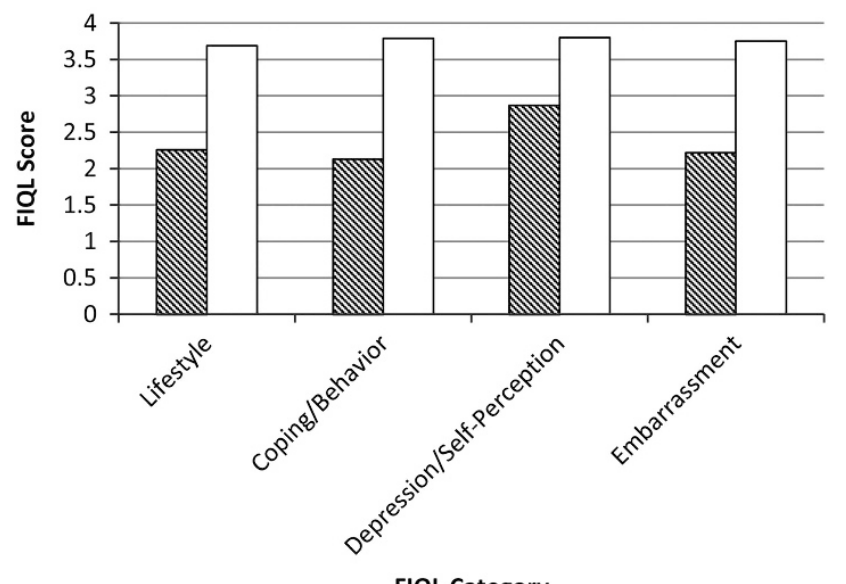

FIQL Category

Figure 1 Comparison of preoperative and postoperative FIQL scores. postoperative assessment was not significantly affected by patient age, gender or level of injury.

The ACE channel was created using appendix in the vast majority of patients (Table 1). In three patients, the appendix was either of insufficient size to create an effective channel or was absent owing to prior appendectomy. In situations in which the appendix could not be used, the ACE channel was created using either ileum or sigmoid colon. Urinary tract reconstruction was performed concurrently with the ACE procedure in four patients. The types of urinary tract reconstructions performed in these patients included creation of a continent catheterizable urinary stoma (Mitrofanoff procedure), bladder augment and bladder neck sling. The timing of the procedure relative to the initial injury was variable among the cohort; however, approximately half of the patients underwent the ACE procedure 10 or more years after their injury.

Four patients developed complications during the follow-up period. Complications included stomal stenosis in three patients and delayed small-bowel obstruction in one patient. There was no association between the type of intestinal segment used for the ACE channel and the development of postoperative complications.

\section{DISCUSSION}

SCI is a devastating condition, which disproportionately affects younger patients with long life expectancy. Neurogenic bowel dysfunction resulting in severe constipation and fecal incontinence is a common consequence of SCI, and it is the source of some of the most bothersome symptoms in this patient population. ${ }^{2,3}$ To our knowledge, no previous study has specifically addressed the impact of the ACE procedure on QOL related to fecal incontinence in patients with neurogenic bowel dysfunction due to SCI. Using a diseasespecific QOL instrument, the FIQL survey, we showed significant improvements in QOL following the ACE procedure in patients with neurogenic bowel dysfunction secondary to SCI. Significant improvements in QOL scores were identified in all four QOL domains assessed by the FIQL survey, with the most marked changes observed in the

Table 1 Clinical and demographic characteristics of patients undergoing the ACE procedure

\begin{tabular}{|c|c|c|c|c|c|}
\hline Gender & $\begin{array}{l}\text { Level of } \\
\text { injury }\end{array}$ & $\begin{array}{l}\text { Age at } \\
\text { surgery }\end{array}$ & $\begin{array}{c}\text { Years } \\
\text { from } S C I \\
\text { to } A C E\end{array}$ & $\begin{array}{c}\text { Appendix } \\
\text { used }\end{array}$ & $\begin{array}{c}\text { Urinary reconstruction } \\
\text { at the time of } A C E\end{array}$ \\
\hline M & T9 & 48 & 19 & Yes & Yes \\
\hline M & T4 & 19 & 13 & Yes & No \\
\hline$F$ & T4 & 44 & 1 & Yes & Yes \\
\hline M & C5 & 43 & 11 & Yes & No \\
\hline M & T4 & 6 & 3 & Yes & No \\
\hline$F$ & C5 & 29 & 2 & Yes & Yes \\
\hline M & C6 & 37 & 17 & Yes & No \\
\hline$F$ & T11 & 22 & 4 & No & No \\
\hline M & T11 & 36 & 6 & Yes & No \\
\hline M & C7 & 49 & 5 & No & No \\
\hline M & $\mathrm{T} 1$ & 13 & 10 & Yes & No \\
\hline $\mathrm{M}$ & C5 & 31 & 11 & Yes & No \\
\hline $\mathrm{M}$ & C5 & 34 & 6 & Yes & No \\
\hline $\mathrm{M}$ & T4 & 48 & 30 & Yes & No \\
\hline $\mathrm{F}$ & C6 & 39 & 12 & Yes & Yes \\
\hline $\mathrm{F}$ & C5 & 37 & 14 & No & No \\
\hline M & T10 & 22 & 6 & Yes & No \\
\hline
\end{tabular}

Abbreviations: ACE, antegrade continence enema; F, female; M, male; SCI, spinal cord injury. 
domains pertaining to patient embarrassment, lifestyle and coping related to fecal incontinence.

In addition to noting significant improvement in FIQL scores following the ACE procedure, we also demonstrated postoperative FIQL scores in our population that approach scores that would be expected among individuals without fecal incontinence. On the continuous scale of $1-4$, where a score of 4 implies no impact of fecal incontinence on QOL, we recognized postoperative FIQL scores ranging from 3.7 to 3.8 among the four QOL domains. Conversely, the low preoperative FIQL scores seen in our series highlight the profound impact of fecal incontinence on QOL in this patient population.

Previous series have shown that fecal incontinence, reliance on a caregiver to assist with toileting and the need for time-consuming bowel regimens are major components of the bowel function-related distress experienced by patients with SCI. ${ }^{15}$ The significant improvements in QOL seen in the present study are likely multifactorial and may be related to improved fecal continence, patient independence with bowel management and a less time-consuming bowel regimen following the ACE procedure.

The ACE procedure has become a mainstay in the treatment of refractory constipation and fecal incontinence in patients with neurogenic bowel dysfunction since its introduction in 1990. Previous series have highlighted a QOL benefit from the ACE procedure in patients with fecal incontinence due to neurogenic bowel dysfunction of various etiologies. Ok and Kurzrock ${ }^{12}$ have developed and validated a QOL questionnaire to assess the impact of fecal incontinence and constipation on the families of children with spina bifida. In their prospective series of 18 families of patients with spina bifida, fecal incontinence and QOL scores improved significantly after the ACE procedure. Laurberg reported long-term outcomes in 69 adult patients who had undergone the ACE procedure, 33 of whom had neurogenic bowel dysfunction. Using an arbitrary scale, patient-reported bowel function, social function and QOL all significantly improved after the ACE procedure (mean follow-up 75 months). ${ }^{7}$

Because SCI is a multifactorial disability, we believe that a diseasespecific QOL instrument, which specifically addresses QOL related to fecal incontinence, is the most appropriate means to measure the QOL impact of the ACE procedure as a procedural outcome in SCI patients. The FIQL instrument not only meets this criterion, but it also correlates with comparable scales of the SF-36 global QOL instrument, which is a widely used measure of QOL in the SCI population. ${ }^{14}$ Although we demonstrated significant improvements in QOL specifically related to fecal incontinence, the impact of the ACE procedure on global QOL in the SCI population remains undefined. As the impact of fecal incontinence is weighed with other global QOL factors, the benefit to global QOL may be less significant. This phenomenon is highlighted by the work of MacNeily et al. ${ }^{16}$ (2009) that showed no global QOL benefit to lower urinary tract reconstruction in the spina bifida population.

Stomal stenosis is one of the most common complications of the ACE procedure. Stomal stenosis requiring revision occurred in $18 \%$ $(3 / 17)$ of our patients, which is comparable to that reported in other series. $^{5-7,17}$ One patient $(6 \%)$ in our series developed a delayed smallbowel obstruction, which required surgical exploration. The risk of major complications following the ACE procedure, although low, underscores the role of proper preoperative counseling.

Our series is limited by a relatively small cohort size, which we attribute to the infrequency with which the ACE procedure is performed in patients with SCI. Larger prospective studies will be needed to fully define the positive QOL impact of the ACE procedure in patients with SCI demonstrated in this pilot study. In addition, the present series selects for patients with more severe symptoms related to bowel dysfunction that have been refractory to conservative measures and may not reflect the impact of fecal incontinence on QOL in the SCI population at large. We believe that the QOL benefit seen in our study objectively highlights the value of the ACE procedure in patients with refractory fecal incontinence due to SCI, and it may aid in proper patient selection and preoperative counseling.

\section{CONCLUSIONS}

The ACE procedure is a highly effective method of treating severe constipation and fecal incontinence in patients with neurogenic bowel dysfunction due to SCI. In our series of 17 SCI patients with neurogenic bowel dysfunction, the ACE resulted in marked improvements in QOL related to fecal incontinence.

\section{DATA ARCHIVING}

There were no data to deposit.

\section{CONFLICT OF INTEREST}

The authors declare no conflict of interest.

1 Han TR, Kim JH, Kwon BS. Chronic gastrointestinal problems and bowel dysfunction in patients with spinal cord injury. Spinal Cord 1998; 36: 485-490.

2 Ng C, Prott G, Rutkowski S, Li Y, Hansen R, Kellow J et al. Gastrointestinal symptoms in spinal cord injury: relationships with level of injury and psychologic factors. Dis Colon Rectum 2005; 48: 1562-1568.

3 Valles M, Vidal J, Clave P, Mearin F. Bowel dysfunction in patients with motor complete spinal cord injury: clinical, neurological, and pathophysiological associations. Am J Gastroenterol 2006; 101: 2290-2299.

4 Malone PS, Ransley PG, Kiely EM. Preliminary report: the antegrade continence enema. Lancet 1990; 336: 1217-1218.

5 Bani-Hani AH, Cain MP, Kaefer M, Meldrum KK, King S, Johnson CS et al. The Malone antegrade continence enema: single institutional review. J Urol 2008; 180 : 1106-1110.

6 Koivusalo AI, Pakarinen MP, Pauniaho SL, Rintala RJ. Antegrade continence enema in the treatment of congenital fecal incontinence beyond childhood. Dis Colon Rectum 2008; 51: 1605-1610.

7 Teichman JM, Zabihi N, Kraus SR, Harris JM, Barber DB. Long-term results for Malone antegrade continence enema for adults with neurogenic bowel disease. Urology 2003; 61: 502-506.

8 Worsoe J, Christensen P, Krogh K, Buntzen S, Laurberg S. Long-term results of antegrade colonic enema in adult patients: assessment of functional results. Dis Colon Rectum 2008; 51: 1523-1528.

9 Dey R, Ferguson C, Kenny SE, Shankar KR, Coldicutt P, Baillie CT et al. After the honeymoon-medium-term outcome of antegrade continence enema procedure.J Pediatr Surg 2003; 38: 65-68.

10 Ellsworth PI, Webb HW, Crump JM, Barraza MA, Stevens PS, Mesrobian HG. The Malone antegrade colonic enema enhances the quality of life in children undergoing urological incontinence procedures. J Urol 1996; 155: 1416-1418.

11 Mattix KD, Novotny NM, Shelley AA, Rescorla FJ. Malone antegrade continence enema (MACE) for fecal incontinence in imperforate anus improves quality of life. Pediatr Surg Int 2007; 23: 1175-1177.

$12 \mathrm{Ok} \mathrm{JH}$, Kurzrock EA. Objective measurement of quality of life changes after ACE Malone using the FICQOL survey. J Pediatr Urol 2011; 7: 389-393.

13 Rockwood TH, Church JM, Fleshman JW, Kane RL, Mavrantonis C, Thorson AG et al. Fecal Incontinence Quality of Life Scale: quality of life instrument for patients with fecal incontinence. Dis Colon Rectum 2000; 43: 9-16.

14 Boakye M, Leigh BC, Skelly AC. Quality of life in persons with spinal cord injury: comparisons with other populations. J Neurosurg Spine 2012; 17: 29-37.

15 Glickman S, Kamm MA. Bowel dysfunction in spinal-cord-injury patients. Lancet 1996; 347: 1651-1653.

16 MacNeily AE, Jafari S, Scott H, Dalgetty A, Afshar K. Health related quality of life in patients with spina bifida: a prospective assessment before and after lower urinary tract reconstruction. J Urol 2009; 182: 1984-1991.

17 Thomas JC, Dietrich MS, Trusler L, DeMarco RT, JCt Pope, Brock JW 3rd et al. Continent catheterizable channels and the timing of their complications. J Urol 2006; 176: $1816-1820$. 